\title{
Taylor Couette instability in disk suspensions
}

\author{
J. J. J. Gillissen* and H. J. Wilson \\ Department of Mathematics, University College London, Gower Street, London, WC1E 6BT, United Kingdom
}

(Received 9 May 2018; published 20 November 2018)

\begin{abstract}
We study the stability of dilute suspensions of spheroids in Taylor Couette flow. We focus on axisymmetric perturbations and on the limiting cases of thin disks and long rods. It is found that in the non-Brownian limit, the rods have a negligible effect on the stability, while the disks are destabilizing. The instability is driven by a tilting of the disks, which draws energy from the base flow into azimuthal velocity fluctuations. The resulting instability mode has a wavelength which is smaller than the unstable Newtonian mode. These findings may serve to understand experiments using clay suspensions in the literature.
\end{abstract}

DOI: 10.1103/PhysRevFluids.3.113903

\section{INTRODUCTION}

Taylor Couette flow (TCF) is the flow between two concentric cylinders. When the outer cylinder is fixed, and when the rotation speed of the inner cylinder $\Omega$ exceeds a critical value, corresponding to a Taylor number

$$
\mathrm{Ta}=\frac{U \Delta R}{v} \sqrt{\frac{\Delta R}{R_{1}}}
$$

of around 41, the flow undergoes a centrifugal instability and develops an array of axisymmetric vortices [1]. Here $v$ is the fluid kinematic viscosity, $U=\Omega R_{1}$ is the velocity of the inner cylinder, $\Delta R=R_{2}-R_{1}$ is the gap width between the cylinders, and $R_{1}$ and $R_{2}$ are the radii of the inner and of the outer cylinder, respectively. A further increase of Ta induces bifurcations into more complicated (wavy, oscillatory, etc.) vortices and eventually into a state of fully developed turbulence. Owing to the connection with turbulence and the tractability by linear stability analysis, TCF is the subject of a vast amount of literature.

There is particular interest in TCF instability for non-Newtonian fluids. For instance, predicting experimentally measured onset conditions for instability provides a stringent test in the development of constitutive models for complex fluids. In this context, most work has focused on solutions of linear polymers. In inertialess flows, polymers induce an elastic instability, which is driven by an inward force that results from the elastic hoop stress [2,3]. This instability counteracts the Newtonian instability, which is driven by the outward centrifugal force [1]. These opposing mechanisms explain how in inertia-elastic flows polymers may either induce a destabilizing effect [3-7] or a stabilizing effect [6,8]. In addition, at higher concentrations, polymer solutions are shear thinning, and the equations for shear thinning fluids predict a destabilization of the centrifugal mode [9].

In this work, we study TCF instability of dilute suspensions of spheroids. Here, dilute means that the fraction of hydrodynamically coupled fluid is smaller than unity. Since the coupled fluid

\footnotetext{
*jurriaangillissen@gmail.com
} 
is contained in the spheroid circumscribing sphere and since the radius of this sphere equals the spheroid semimajor axis $\max (a, b)$, the dilute condition reads

$$
c r_{a}^{-1} \ll 1 \quad \text { if } r_{a} \leqslant 1, \quad c r_{a}^{2} \ll 1 \quad \text { if } r_{a} \geqslant 1 .
$$

Here $c$ is the spheroid volume fraction, $r_{a}=a / b$ is the aspect ratio, $a$ is the polar radius, and $b$ is the equatorial radius. The cases $r_{a}<1, r_{a}=1$, and $r_{a}>1$ correspond to oblate spheroids (disks), spheres, and prolate spheroids (rods), respectively. Previous numerical work on rods shows a $10 \%$ increase of the critical Ta (stabilization) [10], while previous experiments on disks (clay) show a $75 \%$ reduction of the critical Ta (destabilization) [11]. Since the observed stabilizing effect for clay exceeds that of a purely shear thinning fluid, fluid anisotropy is believed to play a role [11].

In this work, we theoretically study the TCF instability of spheroid suspensions. Our main goal is to elucidate the stability behavior in suspensions of disks.

\section{CONSTITUTIVE EQUATIONS}

TCF is described in cylindrical coordinates, $r, \phi, z=1,2,3$, and is governed by the continuity equation

$$
\nabla \cdot u=0
$$

and the momentum equation

$$
\partial_{t} \boldsymbol{u}=\nabla \cdot\left[-\boldsymbol{u} \boldsymbol{u}-p \boldsymbol{\delta}+v\left(\nabla \boldsymbol{u}+\nabla \boldsymbol{u}^{T}\right)+\boldsymbol{\sigma}\right],
$$

where $\boldsymbol{u}$ is the fluid velocity, $p$ is the fluid pressure (divided by the fluid mass density), $v$ is the fluid kinematic viscosity, and $\boldsymbol{\nabla} \boldsymbol{u}$ is the velocity gradient tensor, which in cylindrical coordinates reads

$$
\nabla_{i} u_{j}=\left(\begin{array}{ccc}
\partial_{r} u_{r} & \partial_{r} u_{\phi} & \partial_{r} u_{z} \\
-r^{-1} u_{\phi} & r^{-1} u_{r} & 0 \\
\partial_{z} u_{r} & \partial_{z} u_{\phi} & \partial_{z} u_{z}
\end{array}\right) .
$$

The stress induced by the spheroids reads (see Eq. (4.25) in Ref. [12])

$$
\frac{\boldsymbol{\sigma}}{v}=2 \alpha_{1} \boldsymbol{s}+2 \alpha_{2} \boldsymbol{s}: \boldsymbol{a} \boldsymbol{a}+\alpha_{3}(\boldsymbol{s} \cdot \boldsymbol{a}+\boldsymbol{a} \cdot \boldsymbol{s})+\alpha_{4} D_{r}\left(\boldsymbol{a}-\frac{1}{3} \boldsymbol{\delta}\right),
$$

where $\boldsymbol{a}$ is referred to as the microstructure tensor, $s=\frac{1}{2}\left(\nabla \boldsymbol{u}+\nabla \boldsymbol{u}^{T}\right)$ is the rate of strain tensor, and $D_{r}$ is the rotary diffusivity, whose relative strength is expressed by the Peclet number

$$
\mathrm{Pe}=\frac{U}{D_{r} \Delta R} .
$$

The material constants $\alpha_{i}$ are linear in $c$ and functions of $r_{a}$. These functions are listed in the Appendix and are plotted in Fig. 1. This figure shows that the scaling of the dominant material constants follows

$$
\left|\alpha_{i}\right| \sim\left\{\begin{array}{ll}
c r_{a}^{-1} & \text { if } r_{a} \ll 1 \\
c r_{a}^{2} & \text { if } r_{a} \gg 1
\end{array},\right.
$$

and by comparison with Eq. (2), we see that diluteness requires $\left|\alpha_{i}\right| \ll 1$. This means that the equation for the spheroid stress $\sigma$ [Eq. (5)] is valid only for cases when $\sigma$ is small compared to the Newtonian stress. This suggests that in its validity range Eq. (5) has a small effect on stability. This is indeed observed for rods. For disks, however, we find a surprisingly strong destabilizing effect, even under dilute conditions; see Fig. 5 below.

In Eq. (5), we have made use of the quadratic closure [13] to express the fourth-order moment, $\langle\boldsymbol{n n n n n}\rangle=\int \Psi(\boldsymbol{n}) \boldsymbol{n n n n} d \boldsymbol{n}=\langle\boldsymbol{n n}\rangle\langle\boldsymbol{n n}\rangle$, of the distribution $\Psi$ of the spheroid orientation vector $\boldsymbol{n}$ (Fig. 2), in terms of the second-order moment, $\langle\boldsymbol{n} \boldsymbol{n}\rangle=\int \Psi(\boldsymbol{n}) \boldsymbol{n} \boldsymbol{n} d \boldsymbol{n}$, where the latter is referred 


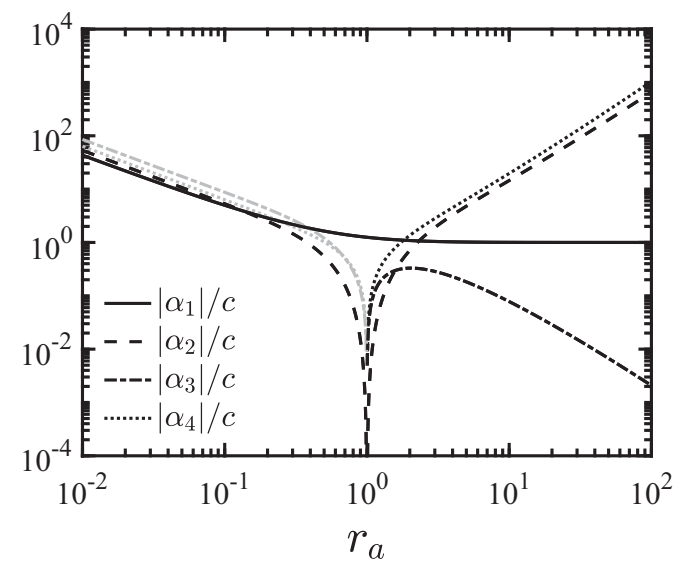

FIG. 1. Absolute values of the dilute spheroid suspension material constants $\alpha_{i}$, divided by the spheroid volume fraction $c$. These quantities are defined in Eq. (5), and they are functions of the aspect ratio $r_{a}$. The relations are taken from Ref. [12] and are listed in the Appendix. The black (gray) lines indicate positive (negative) $\alpha_{i}$.

to as the microstructure tensor, $\boldsymbol{a}=\langle\boldsymbol{n n}\rangle$. The quadratic closure is exact for unidirectional $\Psi$ [13], which is satisfied for thin rods and for thin disks, in the limit of vanishing Brownian motion: $\mathrm{Pe} \gg 1$ [Eq. (6)]. The main finding of the present work concerns this limit (Fig. 6 below), which is therefore not affected by the closure approximation. In the opposite limit of $\mathrm{Pe} \ll 1$, however, the distribution function is isotropic, and the quadratic closure is incorrect. Despite this, the computed stability is not affected by the closure approximation, which correctly approaches the Newtonian limit for Pe $\ll 1$; see Fig. 6 below. For intermediate Pe $\sim$ 1, the authors of Ref. [10] found no significant difference between the quadratic closure and a more sophisticated closure in their TCF stability analysis of rod suspensions.

The microstructure $\boldsymbol{a}$ evolves as (see Eq. (4.26) in Ref. [12])

$$
\partial_{t} \boldsymbol{a}=-\boldsymbol{u} \cdot \nabla \boldsymbol{a}+\nabla \boldsymbol{u}^{T} \cdot \boldsymbol{a}+\boldsymbol{a} \cdot \nabla \boldsymbol{u}+(B-1)(\boldsymbol{s} \cdot \boldsymbol{a}+\boldsymbol{a} \cdot \boldsymbol{s})-2 B \boldsymbol{s}: \boldsymbol{a} \boldsymbol{a}-D_{r}\left(\boldsymbol{a}-\frac{1}{3} \boldsymbol{\delta}\right),
$$

where

$$
B=\frac{r_{a}^{2}-1}{r_{a}^{2}+1}
$$

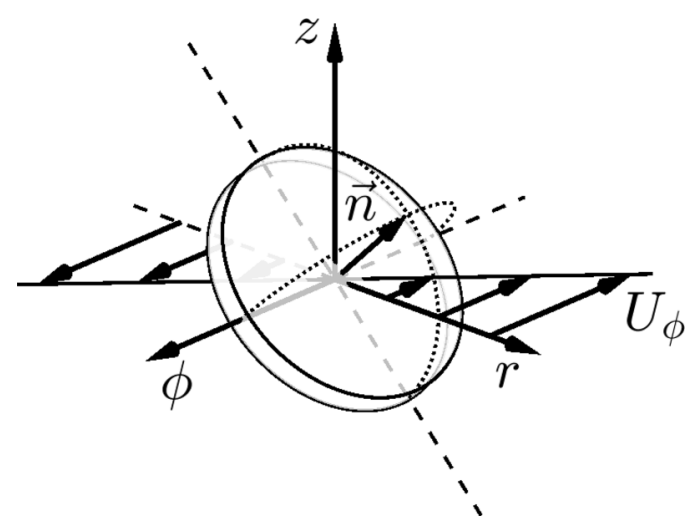

FIG. 2. Coordinate system $r, \phi, z$, base flow field $U_{\phi}(r)$, and particle orientation unit vector $\boldsymbol{n}$. 
In Eq. (8), the cylindrical components of the tensor advection are given by

$$
u_{k} \nabla_{k} a_{i j}=u_{r} \partial_{r} a_{i j}+u_{\phi} r^{-1} b_{i j}+u_{z} \partial_{z} a_{i j}
$$

where $b_{i j}$ is given by

$$
b_{i j}=\left(\begin{array}{ccc}
-2 a_{r \phi} & a_{r r}-a_{\phi \phi} & -a_{\phi z} \\
a_{r r}-a_{\phi \phi} & 2 a_{r \phi} & a_{r z} \\
-a_{\phi z} & a_{r z} & 0
\end{array}\right) .
$$

We compute the stability of the laminar solution to Eqs. (3)-(5) and (8), with regard to perturbations that are harmonic functions of $z$, with a wave number $k$. We restrict the analysis to axisymmetric perturbations. To that end, we decompose flow quantities $\boldsymbol{q}=\boldsymbol{Q}(r)+\boldsymbol{q}^{\prime}(r, t) \exp (i k z)$ into a base state, denoted by a capital letter $\boldsymbol{Q}$, and a perturbation, denoted by a prime $\boldsymbol{q}^{\prime} \exp (i k z)$. We thus write $\boldsymbol{u}=\boldsymbol{U}+\boldsymbol{u}^{\prime} \exp (i k z), \boldsymbol{a}=\boldsymbol{A}+\boldsymbol{a}^{\prime} \exp (i k z), \boldsymbol{\sigma}=\boldsymbol{\Sigma}+\boldsymbol{\sigma}^{\prime} \exp (i k z)$, etc.

The velocity base state $\boldsymbol{U}=U_{\phi} \boldsymbol{e}_{\phi}$ and the microstructure base state $\boldsymbol{A}$ are governed by the azimuthal component of the momentum equation [Eq. (4)],

$$
\left(\partial_{r}+2 r^{-1}\right)\left\{\left[1+\alpha_{1}+2 \alpha_{2} A_{r \phi} A_{r \phi}+\alpha_{3} \frac{1}{2}\left(A_{r r}+A_{\phi \phi}\right)\right] G+\alpha_{4} D_{r} A_{r \phi}\right\}=0,
$$

and by the equations for the $r r, r \phi$, and $\phi \phi$, components of the microstructure equation [Eq. (8)]

$$
\begin{gathered}
{\left[-2 B A_{r \phi} A_{r r}+(B-1) A_{r \phi}\right] G-D_{r}\left(A_{r r}-\frac{1}{3}\right)=0,} \\
{\left[A_{r r}-2 B A_{r \phi} A_{r \phi}+(B-1) \frac{1}{2}\left(A_{r r}+A_{\phi \phi}\right)\right] G-D_{r} A_{r \phi}=0,}
\end{gathered}
$$

and

$$
\left[2 A_{r \phi}-2 B A_{r \phi} A_{\phi \phi}+(B-1) A_{r \phi}\right] G-D_{r}\left(A_{\phi \phi}-\frac{1}{3}\right)=0 .
$$

The $r z$ and $\phi z$ components are zero, the $z z$ component follows from normalization $A_{z z}=1-A_{r r}-$ $A_{\phi \phi}$, and $G$ is defined as

$$
G=\left(\partial_{r}-r^{-1}\right) U_{\phi}
$$

The perturbations are governed by the continuity equation [Eq. (3)]

$$
\left(\partial_{r}+r^{-1}\right) u_{r}^{\prime}+i k u_{z}^{\prime}=0,
$$

and by the linearized momentum equations [Eq. (4)]

$$
\begin{gathered}
\partial_{t} u_{r}^{\prime}=-\partial_{r} p^{\prime}+v\left(\partial_{r}^{2}+r^{-1} \partial_{r}-r^{-2}-k^{2}\right) u_{r}^{\prime}+2 r^{-1} U_{\phi} u_{\phi}^{\prime}+\left(\partial_{r}+r^{-1}\right) \sigma_{r r}^{\prime}+i k \sigma_{z r}^{\prime}-r^{-1} \sigma_{\phi \phi}^{\prime}, \\
\partial_{t} u_{\phi}^{\prime}=v\left(\partial_{r}^{2}+r^{-1} \partial_{r}-r^{-2}-k^{2}\right) u_{\phi}^{\prime}-\left[\left(\partial_{r}+r^{-1}\right) U_{\phi}\right] u_{r}^{\prime}+\left(\partial_{r}+2 r^{-1}\right) \sigma_{r \phi}^{\prime}+i k \sigma_{z \phi}^{\prime},
\end{gathered}
$$

and

$$
\partial_{t} u_{z}^{\prime}=-i k p^{\prime}+v\left(\partial_{r}^{2}+r^{-1} \partial_{r}-k^{2}\right) u_{z}^{\prime}+\left(\partial_{r}+r^{-1}\right) \sigma_{r z}^{\prime}+i k \sigma_{z z}^{\prime},
$$




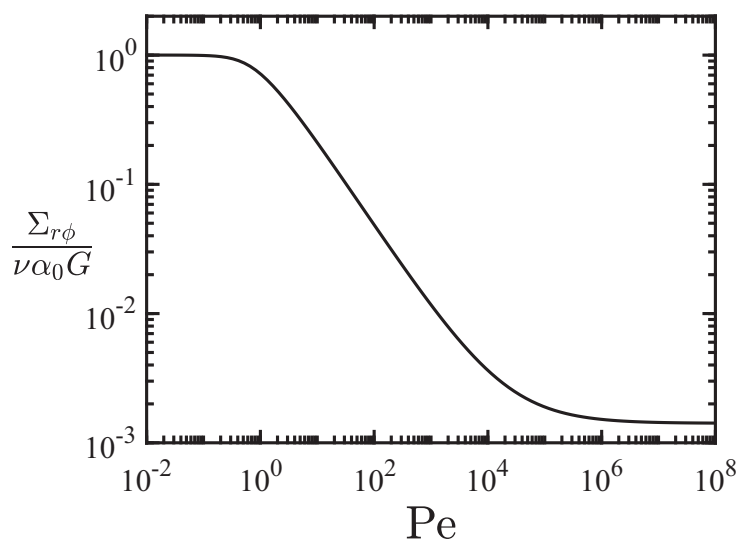

FIG. 3. Spheroid contribution to the effective viscosity $\Sigma_{r \phi} / G$, scaled by the value at zero shear $v \alpha_{0}$ [Eq. (21)], as a function of the Peclet number Pe [Eq. (6)] for disks with $r_{a}=10^{-3}$, computed by solving Eqs. (9)-(12) and (20). The shear rate $G$ is defined in Eq. (13).

and by the linearized microstructure equation [Eq. (8)]

$$
\begin{aligned}
\partial_{t} \boldsymbol{a}^{\prime}= & -u_{r}^{\prime} \partial_{r} \boldsymbol{A} \\
& -u_{\phi}^{\prime} r^{-1}\left(\begin{array}{ccc}
-2 A_{r \phi} & A_{r r}-A_{\phi \phi} & 0 \\
A_{r r}-A_{\phi \phi} & 2 A_{r \phi} & 0 \\
0 & 0 & 0
\end{array}\right)-U_{\phi} r^{-1}\left(\begin{array}{ccc}
-2 a_{r \phi}^{\prime} & a_{r r}^{\prime}-a_{\phi \phi}^{\prime} & -a_{\phi z}^{\prime} \\
a_{r r}^{\prime}-a_{\phi \phi}^{\prime} & 2 a_{r \phi}^{\prime} & a_{r z}^{\prime} \\
-a_{\phi z}^{\prime} & a_{r z}^{\prime} & 0
\end{array}\right) \\
& +\nabla \boldsymbol{u}^{\prime T} \cdot \boldsymbol{A}+\boldsymbol{A} \cdot \nabla \boldsymbol{u}^{\prime}+\nabla \boldsymbol{U}^{T} \cdot \boldsymbol{a}^{\prime}+\boldsymbol{a}^{\prime} \cdot \boldsymbol{\nabla} \boldsymbol{U}-2 B\left(\boldsymbol{S}: \boldsymbol{A} \boldsymbol{a}^{\prime}+\boldsymbol{S}: \boldsymbol{a}^{\prime} \boldsymbol{A}+\boldsymbol{s}^{\prime}: \boldsymbol{A} \boldsymbol{A}\right) \\
& +(B-1)\left(\boldsymbol{S} \cdot \boldsymbol{a}^{\prime}+\boldsymbol{a}^{\prime} \cdot \boldsymbol{S}+\boldsymbol{s}^{\prime} \cdot \boldsymbol{A}+\boldsymbol{A} \cdot \boldsymbol{s}^{\prime}\right)-D_{r} \boldsymbol{a}^{\prime},
\end{aligned}
$$

where the perturbed stress [Eq. (5)] reads

$$
\begin{aligned}
\frac{\boldsymbol{\sigma}^{\prime}}{v}= & 2 \alpha_{1} \boldsymbol{s}^{\prime}+2 \alpha_{2}\left(\boldsymbol{S}: \boldsymbol{A} \boldsymbol{a}^{\prime}+\boldsymbol{S}: \boldsymbol{a}^{\prime} \boldsymbol{A}+\boldsymbol{s}^{\prime}: \boldsymbol{A} \boldsymbol{A}\right) \\
& +\alpha_{3}\left(\boldsymbol{S} \cdot \boldsymbol{a}^{\prime}+\boldsymbol{a}^{\prime} \cdot \boldsymbol{S}+\boldsymbol{s}^{\prime} \cdot \boldsymbol{A}+\boldsymbol{A} \cdot \boldsymbol{s}^{\prime}\right)+\alpha_{4} D_{r} \boldsymbol{a}^{\prime} .
\end{aligned}
$$

\section{EFFECTIVE VISCOSITY}

The base state is a shear flow with an $r$-dependent shear rate $G$ [Eq. (13)]. The ratio of the shear stress $v G+\Sigma_{r \phi}$ and $G$ is referred to as the effective viscosity: $v_{\text {eff }}=v+\Sigma_{r \phi} / G$. This quantity is found by inserting the corresponding velocity gradient

$$
\nabla_{i} U_{j}=\left(\begin{array}{ccc}
0 & \partial_{r} U_{\phi} & 0 \\
-r^{-1} U_{\phi} & 0 & 0 \\
0 & 0 & 0
\end{array}\right)
$$

into the unperturbed version of the $r \phi$ component of Eq. (5),

$$
\frac{v_{\mathrm{eff}}}{v}=1+\alpha_{1}+2 \alpha_{2} A_{r \phi}^{2}+\frac{\alpha_{3}}{2}\left[A_{r r}+A_{\phi \phi}\right]+\alpha_{4} D_{r} A_{r \phi} G^{-1},
$$

where $A_{r r}, A_{r \phi}$, and $A_{\phi \phi}$, are governed by Eqs. (10)-(12). The spheroid contribution to the effective viscosity is plotted for disks in Fig. 3, as a function of the Peclet number Pe [Eq. (6)]. For Pe $\ll 1$, the dynamics is dominated by Brownian motion and the viscosity is shear rate independent. The spheroid contribution to this so-called zero-shear rate viscosity is denoted $\alpha_{0}$ and is found from 
Eqs. (10)-(12) and (20):

$$
\alpha_{0}=\frac{\Sigma_{r \phi}}{G v}=\frac{\alpha_{1}+B \alpha_{4}}{3} .
$$

For Pe $\gg 1$, the disks align their normals in the gradient $(r)$ direction, which results in the shear thinning behavior in Fig. 3. Rods produce a similar shear thinning behavior (not shown).

In the present work, we consider dilute suspensions $\alpha_{0} \lesssim 1$ [Eq. (21)]. As an effect, the spheroid contribution to the effective viscosity is small, which is therefore only mildly shear thinning.

\section{ANALYTICAL SOLUTION}

\section{A. Approximations}

Before numerically studying the TCF instability, we first employ a few simplifications, allowing a transparent, analytical solution and thereby providing insight into the stability behavior for some limiting cases. To this end, we consider the limit of the infinitesimal gap $\Delta R / R_{1} \ll 1$, such that $\partial_{r} \gg r^{-1}$. We furthermore approximate

$$
\partial_{r} u_{r}^{\prime}=i l u_{r}^{\prime}, \quad \partial_{r} u_{z}^{\prime}=i l u_{z}^{\prime}, \quad \partial_{r} u_{\phi}^{\prime}=i m u_{\phi}^{\prime},
$$

where $l$ is the radial wave number for the transverse velocity fluctuations $\left(u_{r}^{\prime}, u_{z}^{\prime}\right)$ and $m$ is the radial wave number for the azimuthal velocity fluctuations $u_{\phi}^{\prime}$. As shown in Fig. 8, the most unstable mode corresponds to $l \approx 2 \pi / \Delta R$ and $m \approx \pi / \Delta R$. In addition, the velocity in the gap $U_{\phi}=U\left(R_{2}-r\right) / \Delta R$ and its radial derivative are approximated by

$$
U_{\phi}(r)=\frac{U}{2} \quad \partial_{r} U_{\phi}(r)=-\frac{U}{\Delta R} .
$$

\section{B. Newtonian}

Under the approximations listed in Sec. IV A, Eqs. (14)-(17) reduce to

$$
\begin{gathered}
i l u_{r}^{\prime}+i k u_{z}^{\prime}=0, \\
\partial_{t} u_{\phi}^{\prime}=\frac{U}{\Delta R} u_{r}^{\prime}-v\left(m^{2}+k^{2}\right) u_{\phi}^{\prime},
\end{gathered}
$$

and

$$
\partial_{t}\left(i k u_{r}^{\prime}-i l u_{z}^{\prime}\right)=i k\left[\frac{U}{R} u_{\phi}^{\prime}-v\left(l^{2}+k^{2}\right) u_{r}^{\prime}\right]+v i l\left(l^{2}+k^{2}\right) u_{z}^{\prime},
$$

where $R \approx R_{1} \approx R_{2}$, and we have eliminated the pressure $p$ by combining Eqs. (15) and (17) into Eq. (26). Equations (24) and (26) can be combined to give

$$
\partial_{t} u_{r}^{\prime}=\frac{U}{\left[1+\left(\frac{l}{k}\right)^{2}\right] R} u_{\phi}^{\prime}-v\left(l^{2}+k^{2}\right) u_{r}^{\prime} .
$$

By writing Eqs. (25) and (27) as

$$
\partial_{t}\left(\begin{array}{c}
u_{r}^{\prime} \\
u_{\phi}^{\prime}
\end{array}\right)=\left(\begin{array}{cc}
-v\left(l^{2}+k^{2}\right) & \frac{U}{\left[1+\left(\frac{l}{k}\right)^{2}\right]_{R}} \\
\frac{U}{\Delta R} & -v\left(m^{2}+k^{2}\right)
\end{array}\right)\left(\begin{array}{l}
u_{r}^{\prime} \\
u_{\phi}^{\prime}
\end{array}\right),
$$

the perturbation growth rates $\lambda$ are found by computing the eigenvalues of the matrix in Eq. (28). The maximum growth rate is real valued, i.e., nonoscillatory. The flow becomes unstable, when the maximum growth rate passes through zero. The corresponding Taylor number [Eq. (1)] is plotted in Fig. 4(a) as a function of the spanwise wave number $k$. The most unstable mode corresponds 

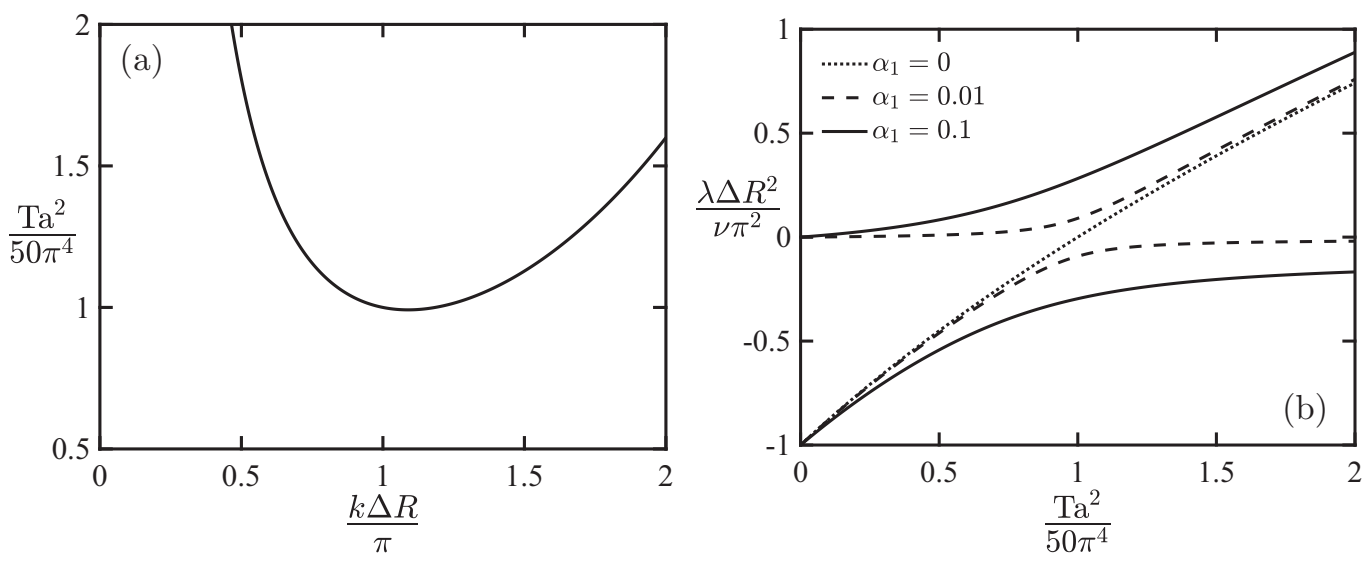

FIG. 4. (a) Taylor number Ta [Eq. (1)] in Newtonian flow, for which the maximum of the growth rates [eigenvalue of matrix in Eq. (28)] equals zero, as a function of the spanwise wave number $k$. (b) Growth rates $\lambda$ [eigenvalues of matrix in Eq. (32)] as a function of the Taylor number Ta [Eq. (1)] for various concentrations $\alpha_{1}$ of non-Brownian disks, using $k \Delta R / \pi=1$.

to the minimum of this function, which is found at a critical wave number of $k \Delta R / \pi \approx 1$, in agreement with the exact result [1]. The corresponding so-called critical Taylor number equals $\mathrm{Ta} \approx \pi^{2} \sqrt{50} \approx 70$. The discrepancy with the exact result, Ta $\approx 41$ [1], is due to the approximations given by Eqs. (22) and (23). Nevertheless, the simplified model [Eq. (28)] predicts the correct scaling relationship between the governing parameters at the critical point.

\section{Rods}

Non-Brownian rods are governed by the limits $\mathrm{Pe} \rightarrow \infty$ and $r_{a} \rightarrow \infty$, such that $D_{r}=\alpha_{1}=$ $\alpha_{3}=0$ and $B=1$. From Eqs. (10)-(12), we find the base microstructure $\boldsymbol{A}=\boldsymbol{e}_{\phi} \boldsymbol{e}_{\phi}$, which corresponds to zero base stress: $\boldsymbol{\Sigma}=\mathbf{0}$. From Eq. (18), it follows that the microstructure tensor is neutrally stable $\partial_{t} \boldsymbol{a}^{\prime}=\mathbf{0}$, and the perturbed, spheroid stress [Eq. (19)] becomes

$$
\boldsymbol{\sigma}^{\prime}=2 v \alpha_{2} r^{-1} u_{r}^{\prime} \boldsymbol{e}_{\phi} \boldsymbol{e}_{\phi}
$$

which in the limit of infinitesimal gap $\Delta R / R \ll 1$ scales as $\sim \alpha_{2} v u_{r}^{\prime} / R$. Under dilute conditions $\alpha_{2} \lesssim 1$, this stress is negligibly small, compared to the Newtonian viscous stress, which scales as $\sim v u_{r}^{\prime} / \Delta R$. Non-Brownian rods have therefore a negligible effect on the stability in the infinitesimal gap limit: $\Delta R / R \ll 1$.

\section{Disks}

Non-Brownian disks are governed by the limits $\mathrm{Pe} \rightarrow \infty$ and $r_{a} \rightarrow 0$, such that $D_{r}=0, B=$ -1 , and $\alpha_{3}=-2 \alpha_{1}$. From Eqs. (10)-(12), we find the base microstructure $\boldsymbol{A}=\boldsymbol{e}_{r} \boldsymbol{e}_{r}$, which corresponds to zero base stress: $\boldsymbol{\Sigma}=\mathbf{0}$. From Eqs. (18) and (19), it follows that

$$
\partial_{t} \boldsymbol{a}^{\prime}=-i k u_{r}^{\prime} \boldsymbol{e}_{r} \boldsymbol{e}_{z}
$$

and

$$
\boldsymbol{\sigma}^{\prime}=2 v\left(\alpha_{2}-\alpha_{1}\right)\left(\begin{array}{ccc}
s_{r r}^{\prime} & 0 & 0 \\
0 & 0 & 0 \\
0 & 0 & 0
\end{array}\right)+2 v \alpha_{1}\left(\begin{array}{ccc}
0 & 0 & 0 \\
0 & s_{\phi \phi}^{\prime} & s_{\phi z}^{\prime} \\
0 & s_{\phi z}^{\prime} & s_{z z}^{\prime}
\end{array}\right)+a_{r z}^{\prime} v \alpha_{1} \frac{U}{\Delta R}\left(\begin{array}{ccc}
0 & 0 & 0 \\
0 & 0 & 1 \\
0 & 1 & 0
\end{array}\right) .
$$


When the growth rate $\lambda$ is sufficiently small, $\lambda \Delta R / U \ll 1$, the third term of Eq. (31) dominates, since $a_{r z}^{\prime}=-i k u_{r}^{\prime} / \lambda$ [Eq. (30)] and $\alpha_{2} \sim \alpha_{1}$ (Fig. 1). Adding Eq. (30) and the third term of Eq. (31) to the equations of motion [Eq. (28)] gives

$$
\partial_{t}\left(\begin{array}{c}
u_{r}^{\prime} \\
u_{\phi}^{\prime} \\
a_{r z}^{\prime}
\end{array}\right)=\left(\begin{array}{ccc}
-v\left(l^{2}+k^{2}\right) & \frac{U}{\left[1+\left(\frac{l}{k}\right)^{2}\right]_{R}} & 0 \\
\frac{U}{\Delta R} & -v\left(m^{2}+k^{2}\right) & i k v \alpha_{1} \frac{U}{\Delta R} \\
-i k & 0 & 0
\end{array}\right)\left(\begin{array}{c}
u_{r}^{\prime} \\
u_{\phi}^{\prime} \\
a_{r z}^{\prime}
\end{array}\right) .
$$

The two largest growth rates [eigenvalues of the matrix in Eq. (32)] are real valued, i.e., nonoscillatory and are plotted in Fig. 4(b) as a function of the Taylor number Ta [Eq. (1)], using $k \Delta R / \pi=1$ [Fig. 4(a)]. For Newtonian flow $\left(\alpha_{1}=0\right)$, the largest eigenvalue changes from negative to positive, and instability sets in at $\mathrm{Ta} \approx \pi^{2} \sqrt{50}$. For non-Newtonian flow $\left(\alpha_{1}>0\right)$, on the other hand, the matrix always has a positive eigenvalue that vanishes when $\mathrm{Ta} \rightarrow 0$. This result predicts that non-Brownian disks destabilize TCF even at dilute conditions.

\section{NUMERICAL SOLUTION}

We numerically solve the $r$-dependent base state [Eqs. (9)-(12)] and the perturbations [Eqs. (14)(19)] using Chebyshev discretization on 30 collocation points [14]. We consider disks $r_{a}=10^{-3}$ and rods $r_{a}=10^{3}$ and fix the Couette cell radius ratio to $R_{2} / R_{1}=1.05$, which was also used in the experimental stability analysis of Ref. [11] and in the numerical stability analysis of Ref. [10].

The base state $\boldsymbol{Q}=\left(U_{\phi}, A_{r r}, A_{r \phi}, A_{\phi \phi}\right)$ is governed by the $\phi$ component of the momentum equation [Eq. (9)] and the $r r, r \phi$, and $\phi \phi$ components of the microstructure equation [Eqs. (10)(12)]. We denote these four equations as

$$
\boldsymbol{N}(\boldsymbol{Q})=\mathbf{0}
$$

and we solve them using Newton-Raphson iteration

$$
\boldsymbol{Q}^{n+1}=\boldsymbol{Q}^{n}-\omega\left(\frac{\delta \boldsymbol{N}}{\delta \boldsymbol{Q}}\right)^{-1} \boldsymbol{Q}^{n},
$$

where $n$ is the iteration step and the iteration parameter is chosen as $\omega=0.1$. The linearized equations of motion $(\delta \boldsymbol{N} / \delta \boldsymbol{Q}) \delta \boldsymbol{Q}$ read for the azimuthal momentum

$$
\begin{aligned}
& \left(\partial_{r}+2 r^{-1}\right)\left\{\left[1+\alpha_{1}+2 \alpha_{2} A_{r \phi} A_{r \phi}+\alpha_{3} \frac{1}{2}\left(A_{r r}+A_{\phi \phi}\right)\right] \delta G\right. \\
& \left.\quad+\left[4 \alpha_{2} A_{r \phi} \delta A_{r \phi}+\alpha_{3} \frac{1}{2}\left(\delta A_{r r}+\delta A_{\phi \phi}\right)\right] G+\alpha_{4} D_{r} \delta A_{r \phi}\right\}=0
\end{aligned}
$$

and for the microstructure

$$
\begin{gathered}
{\left[-2 B A_{r \phi} A_{r r}+(B-1) A_{r \phi}\right] \delta G} \\
+\left[-2 B\left(\delta A_{r \phi} A_{r r}+A_{r \phi} \delta A_{r r}\right)+(B-1) \delta A_{r \phi}\right] G-D_{r} \delta A_{r r}=0, \\
{\left[A_{r r}-2 B A_{r \phi} A_{r \phi}+(B-1) \frac{1}{2}\left(A_{r r}+A_{\phi \phi}\right)\right] \delta G} \\
+\left[\delta A_{r r}-2 B\left(\delta A_{r \phi} A_{r \phi}+A_{r \phi} \delta A_{r \phi}\right)+(B-1) \frac{1}{2}\left(\delta A_{r r}+\delta A_{\phi \phi}\right)\right] G-D_{r} \delta A_{r \phi}=0,
\end{gathered}
$$

and

$$
\begin{aligned}
& {\left[2 A_{r \phi}-2 B A_{r \phi} A_{\phi \phi}+(B-1) A_{r \phi}\right] \delta G} \\
& \quad+\left[2 \delta A_{r \phi}-2 B\left(\delta A_{r \phi} A_{\phi \phi}+A_{r \phi} \delta A_{\phi \phi}\right)+(B-1) \delta A_{r \phi}\right] G-D_{r} \delta A_{\phi \phi}=0
\end{aligned}
$$




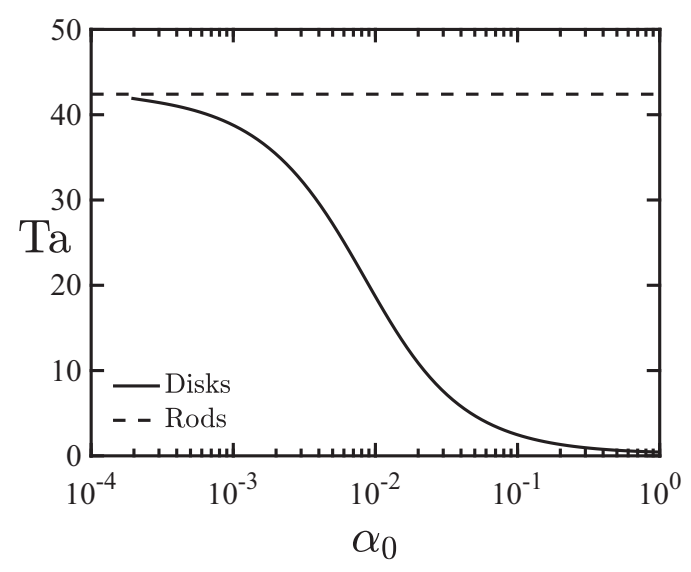

FIG. 5. The critical Taylor number Ta [Eq. (40)] as a function of the concentration parameter $\alpha_{0}$ [Eq. (21)], for disks with an aspect ratio of $r_{a}=10^{-3}$ (solid line) and for rods with an aspect ratio of $r_{a}=10^{3}$ (dashed line), and using a Peclet number [Eq. (6)] of $\mathrm{Pe}=10^{9}$, a spanwise wave number of $k=\pi / \Delta R$, and a radius ratio of $R_{2} / R_{1}=1.05$.

The perturbations $\boldsymbol{q}^{\prime}=\left(\boldsymbol{u}^{\prime}, p^{\prime}, \boldsymbol{a}^{\prime}\right)$ are governed by Eqs. (14)-(19). These equations including the boundary conditions $\left(\boldsymbol{u}^{\prime}=\mathbf{0}\right.$ on the walls) are written in matrix form,

$$
\boldsymbol{M}_{1} \cdot \partial_{t} \boldsymbol{q}^{\prime}=\boldsymbol{M}_{2} \cdot \boldsymbol{q}^{\prime}
$$

and the growth rates are found by solving the corresponding generalized eigenvalue problem in FORTRAN using the ZGGEV routine from the LAPACK library. For all cases discussed below, the eigenvalue of the most unstable mode was found to be real valued, i.e., nonoscillatory.

The rotation rate $\Omega$ is varied to find the onset of instability, which correspond to a sign change of the largest eigenvalue. The onset point is expressed by the effective Taylor number,

$$
\mathrm{Ta}=\frac{\Omega \sqrt{\Delta R^{3} R_{1}}}{v_{\text {eff }}},
$$

where the effective viscosity [Eq. (20)] is evaluated at $r=R_{1}$. The (effective) Taylor number is related to the (effective) Reynolds number $\operatorname{Re}=\Omega R_{1} \Delta R / v_{\text {eff }}$, via $\mathrm{Ta}=\operatorname{Re} \sqrt{\Delta R / R_{1}}$.

In the absence of spheroids, we find a critical Taylor number of Ta $\approx 42$, at a spanwise wave number of $k \approx \pi / \Delta R$. This Ta value is in agreement with the literature, for a gap ratio of $R_{2} / R_{1}=$ 1.05 , which is slightly larger than the value for an infinitesimal gap, Ta $\approx 41$ [15].

Figure 5 shows the critical Taylor number Ta [Eq. (40)] for disks and for rods as a function of the concentration parameter $\alpha_{0}$ [Eq. (21)], using a spanwise wave number of $k=\pi / \Delta R$ and a Peclet number [Eq. (6)] of $\mathrm{Pe}=10^{9}$. As explained in Sec. III, the concentration parameter $\alpha_{0}$ is defined as the spheroid contribution to the relative, zero-shear rate viscosity [Eq. (21)]. For disks $\alpha_{0} \sim c r_{a}^{-1}$ (see the Appendix), and the condition of diluteness [Eq. (2)] is therefore satisfied for $\alpha_{0} \ll 1$. In Fig. 5, we consider the range $10^{-3} \leqslant \alpha_{0} \leqslant 1$, and for the cases where $\alpha_{0} \sim 1$, hydrodynamic interactions are expected to play a role. The corresponding results can therefore only be considered qualitatively correct. For $\alpha_{0} \gtrsim 1$, the numerical method produces unphysical results, where the velocity and microstructure profiles contain spurious waves with a wavelength of two grid spacings (not shown).

Figure 5 shows that for very small concentrations, $\alpha_{0}<10^{-3}$, both disks and rods have no effect on stability; i.e., the critical Taylor number is at the Newtonian value: Ta $\approx 42$. For larger but still dilute concentrations, $10^{-3}<\alpha_{0}<1$, rods remain ineffective. This result is explained in Sec. IV C, showing that under dilute conditions, $\alpha_{0} \lesssim 1$, the perturbed stress of non-Brownian rods 


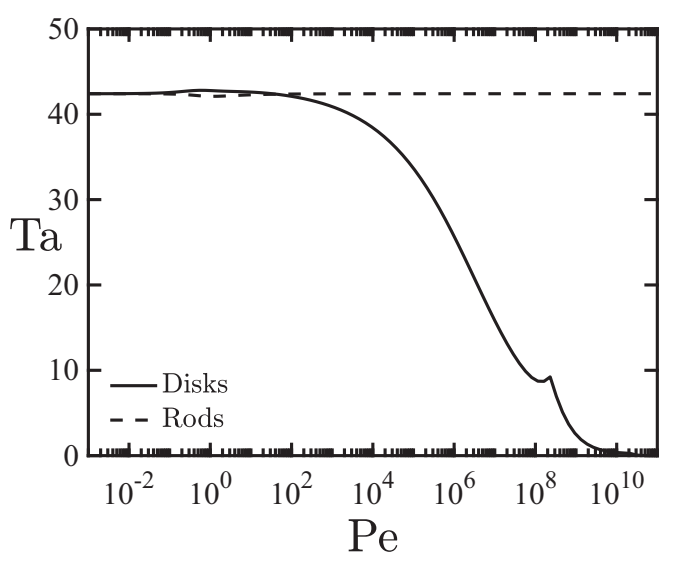

FIG. 6. The critical Taylor number Ta [Eq. (40)] as a function of the Peclet number Pe [Eq. (6)] for disks with an aspect ratio of $r_{a}=10^{-3}$ (solid line) and for rods with an aspect ratio of $r_{a}=10^{3}$ (dashed line), and a concentration parameter [Eq. (21)] of $\alpha_{0}=0.1$, using a spanwise wave number of $k=\pi / \Delta R$ and a radius ratio of $R_{2} / R_{1}=1.05$.

is negligibly small compared to the perturbed solvent stress. Disks, on the other hand, are strongly destabilizing, even under dilute conditions. For instance, for $\alpha_{0}=10^{-2}$, the critical Taylor number is reduced by a factor of two.

Figure 6 shows the critical Taylor number Ta [Eq. (40)] as a function of the Peclet number Pe [Eq. (6)] for disks and for rods. Here we have used a concentration parameter of $\alpha_{0}=0.1$ [Eq. (21)], and a spanwise wave number of $k=\pi / \Delta R$. In the Brownian limit, $\mathrm{Pe} \ll 1$, the microstructure is isotropic and both suspensions are therefore Newtonian, with a critical Taylor number based on the effective viscosity $v_{\text {eff }}$ [Eq. (20)] of $\mathrm{Ta} \approx 42$. In the non-Brownian limit, $\mathrm{Pe} \gg 1$, the rods have a negligible effect on stability, which is explained in Sec. IV C. Non-Brownian disks, on the other hand, are increasingly destabilizing, i.e., $\mathrm{Ta} \rightarrow 0$, as $\mathrm{Pe} \rightarrow \infty$, in agreement with the analysis presented in Sec. IV D. In the intermediate regime $\mathrm{Pe} \sim 1$, the model predicts a few percent increase in Ta for disks (stabilizing) and a few percent decrease in Ta for rods (destabilizing). The intermediate regime $\mathrm{Pe} \sim 1$ is further analyzed in Sec. VI. We also note the ripple in the disk curve at $\mathrm{Pe} \approx 3 \times 10^{8}$. This feature does not seem to be a numerical artefact, as an identical curve was obtained by increasing the number of collocation points from 30 to 40 (not shown).

Figure 7 shows the critical Taylor number Ta [Eq. (40)] as a function of the spanwise wave number $k \Delta R / \pi$ for disks and for rods. Here we have used a Peclet number of $\mathrm{Pe}=10^{9}$ [Eq. (6)] and a concentration parameter of $\alpha_{0}=3 \times 10^{-3}$ [Eq. (21)]. The suspension of rods behaves essentially Newtonian, with a minimum critical Taylor number of $\mathrm{Ta} \approx 42$ at $k \Delta R / \pi \approx 1$, in agreement with literature [1]. For disks, the minimum critical Taylor number is smaller and occurs at a larger wave number, which for this case is $k \Delta R / \pi \approx 1.4$. This result is consistent with experimental observation of a reduced size of the Taylor vortices in clay suspensions [11].

Figure 8 shows the velocity and microstructure components of the most unstable mode, in a suspension of rods, using a Peclet number [Eq. (6)] of $\mathrm{Pe}=10^{9}$, a concentration parameter [Eq. (21)] of $\alpha_{0}=0.1$, a spanwise wave number of $k=\pi / \Delta R$, and a Taylor number [Eq. (40)] at the critical value of $\mathrm{Ta} \approx 42$. Under these conditions, the suspension is essentially Newtonian (see Fig. 5), with a negligibly small perturbed microstructure, and a velocity perturbation, which is dominated by the azimuthal component, in agreement with previous theoretical work [1].

Figure 9 shows the velocity and microstructure components of the most unstable mode, in a suspension of disks, using a Peclet number [Eq. (6)] of $\mathrm{Pe}=10^{9}$, a concentration parameter [Eq. (21)] of $\alpha_{0}=0.1$, a spanwise wave number of: $k=\pi / \Delta R$, and a Taylor number [Eq. (40)] at the critical value of $\mathrm{Ta} \approx 2.5$. It is seen that the cross-stream velocity perturbations are dampened, 


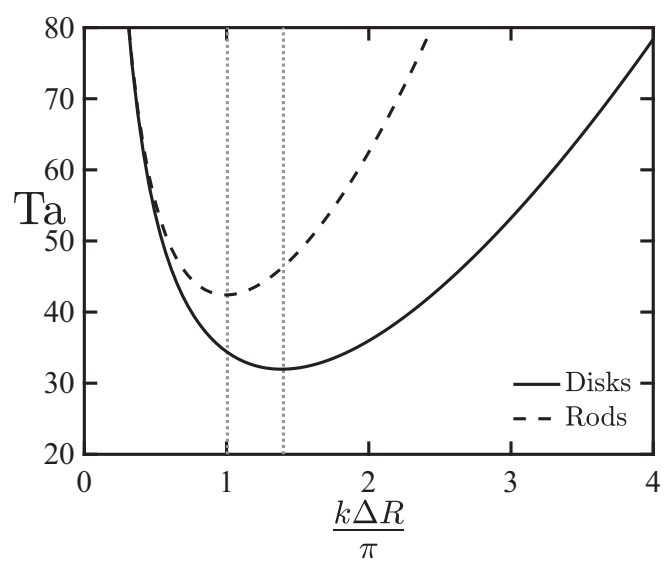

FIG. 7. The critical Taylor number Ta [Eq. (40)] as a function of the spanwise wave number, $k=\pi / \Delta R$, for disks with an aspect ratio of $r_{a}=10^{-3}$ (solid line) and for rods with an aspect ratio of $r_{a}=10^{3}$ (dashed line), a Peclet number [Eq. (6)] of Pe $=10^{9}$, a concentration parameter [Eq. (21)] of $\alpha_{0}=3 \times 10^{-3}$, and a radius ratio of $R_{2} / R_{1}=1.05$. The vertical, dotted lines indicate the minima of the curves.

and there is a significant microstructure perturbation, which is dominated by the $r z$ component. These findings agree with the analysis presented in Sec. IV D.

\section{COMPARISON TO LITERATURE}

Figure 10 compares our results (line) to those of Ref. [10] (markers). The authors of Ref. [10] conducted numerical stability analysis for TCF of suspensions of rods in semidilute conditions, $\alpha_{0} \gtrsim 1$. In Ref. [10], interactions between the rods are accounted for, with a shear-rate-dependent rotary diffusivity [16]

$$
D_{r}=6 C_{I} \dot{\gamma}
$$

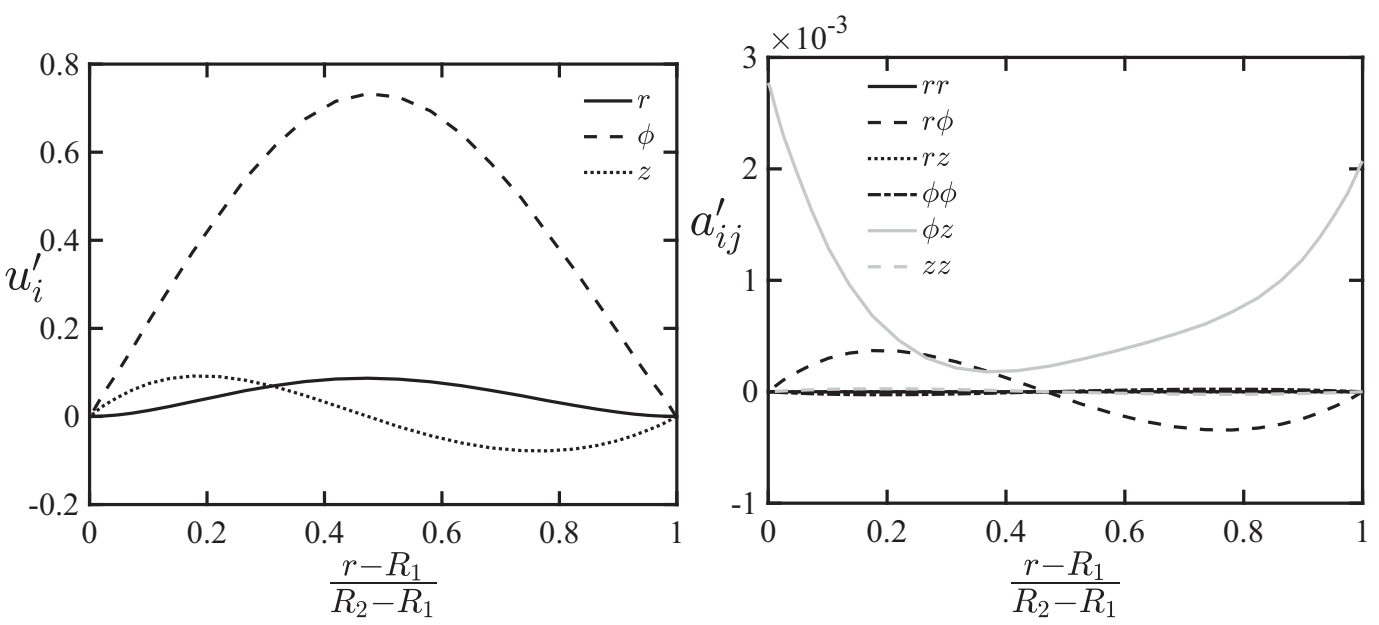

FIG. 8. Most unstable eigenmode in a suspension of rods, using an aspect ratio of $r_{a}=10^{3}$, a Peclet number [Eq. (6)] of Pe $=10^{9}$, a concentration parameter [Eq. (21)] of $\alpha_{0}=0.1$, a spanwise wave number of $k=$ $\pi / \Delta R$, a radius ratio of $R_{2} / R_{1}=1.05$, and a Taylor number [Eq. (40)] at the critical value of $\mathrm{Ta} \approx 42$. 

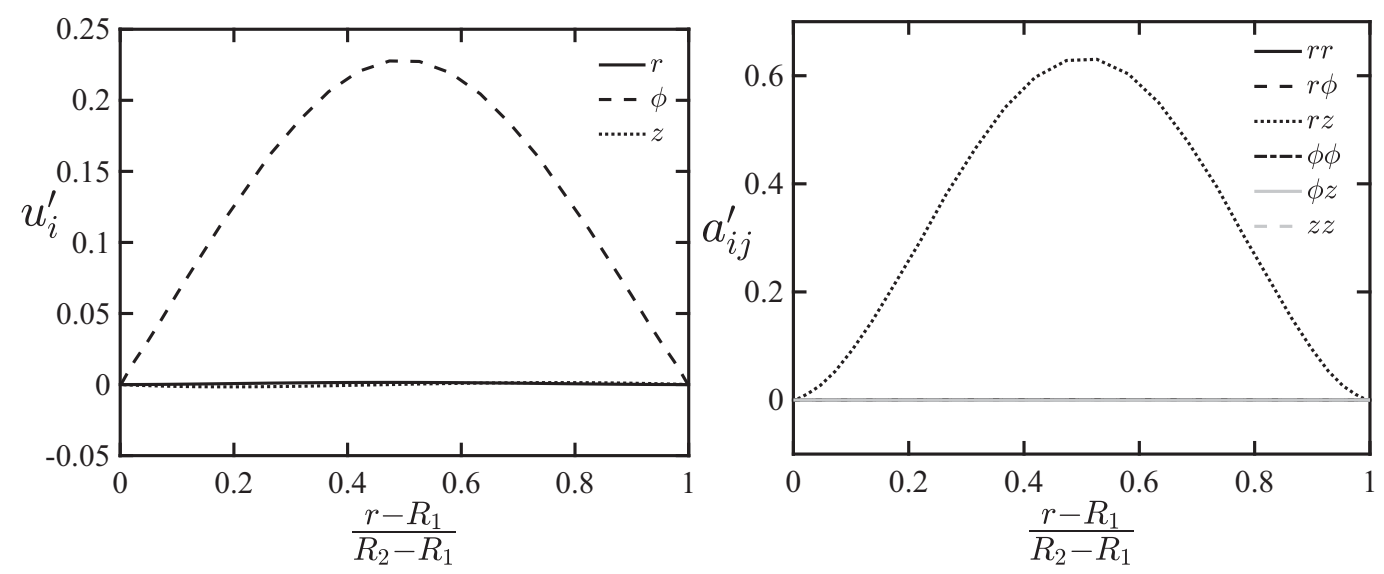

FIG. 9. Most unstable eigenmode in a suspension of disks, using an aspect ratio of $r_{a}=10^{-3}$, a Peclet number [Eq. (6)] of $\mathrm{Pe}=10^{9}$, a concentration parameter [Eq. (21)] of $\alpha_{0}=0.1$, a spanwise wave number of $k=\pi / \Delta R$, a radius ratio of $R_{2} / R_{1}=1.05$, and a Taylor number [Eq. (40)] at the critical value of Ta $\approx 2.5$.

where $C_{I}$ is referred to as the interaction strength and $\dot{\gamma}=\sqrt{2 e: e}$ is the scalar magnitude of the strain-rate tensor $\boldsymbol{e}=\frac{1}{2}\left(\boldsymbol{\nabla} \boldsymbol{u}+\nabla \boldsymbol{u}^{T}\right)$. The present model, on the other hand, does not account for interactions between the spheroids and employs a shear-rate invariant rotary diffusivity. Judging from their Fig. 11, the authors of Ref. [10] also seem to include a spatial diffusion term in the microstructure equation, and enforce $\boldsymbol{a}^{\prime}=\mathbf{0}$ on the walls. These aspects are different in the present model, which does not include spatial diffusion and associated boundary conditions for $\boldsymbol{a}^{\prime}$ (Fig. 8).

The comparison in Fig. 10 concerns rods with an aspect ratio of $r_{a}=10^{3}$ in a Taylor Couete cell with a radius ratio of $R_{2} / R_{1}=1.05$ and a spanwise wave number of $k=\pi / \Delta R$. The rod volume fraction equals $\phi=10^{-4}$, which corresponds to a concentration parameter of $\alpha_{0}=2.35$ [see Eq. (21) and the Appendix], and the interaction strength is varied between $5 \times 10^{-3} \leqslant C_{I} \leqslant 0.2$, which corresponds to a Peclet number $\mathrm{Pe}=1 /\left(6 C_{I}\right)$ of $0.8 \leqslant \mathrm{Pe} \leqslant 33$.

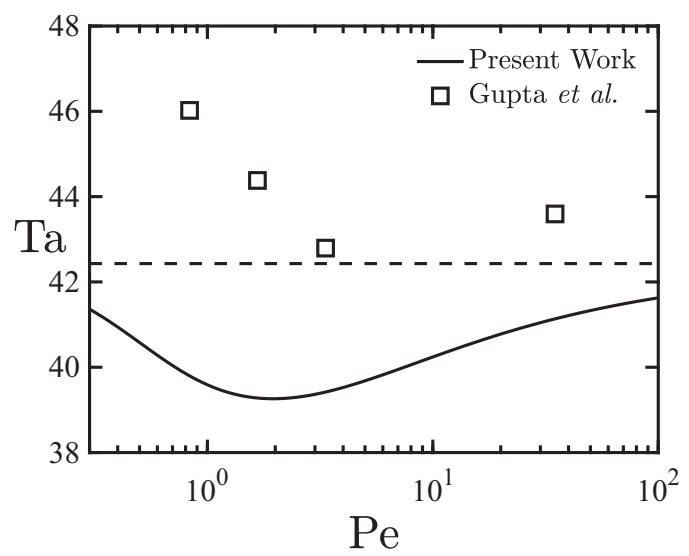

FIG. 10. The critical Taylor number Ta [Eq. (40)] as a function of the Peclet number Pe [Eq. (6)], for rods with an aspect ratio of $r_{a}=10^{3}$, using a concentration parameter [Eq. (21)] of $\alpha_{0}=2.35$, a spanwise wave number of $k=\pi / \Delta R$, and a radius ratio of $R_{2} / R_{1}=1.05$. Comparison between present result (line) and results from Ref. [10] (markers). The dashed line indicates the Newtonian value of Ta $\approx 42.4$. 


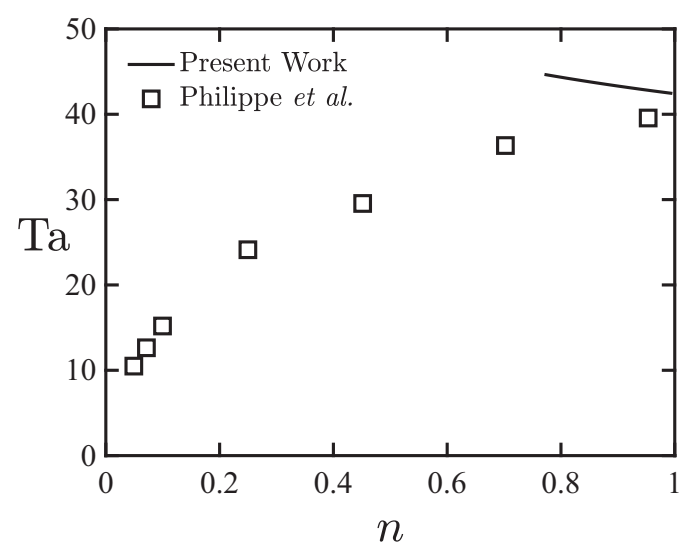

FIG. 11. The critical Taylor number Ta [Eq. (40)] as a function of the fluid index $n$ [Eq. (43)], for disks with an aspect ratio of $r_{a}=3.1 \times 10^{-3}$, using a Peclet number [Eq. (6)] of $\mathrm{Pe}=1.4$, a spanwise wave number of $k=\pi / \Delta R$, and a radius ratio of $R_{2} / R_{1}=1.05$. Comparison between present result (solid line) and results from Ref. [11] (markers).

As shown in Fig. 10, the present model predicts a small $(<10 \%)$ reduction in Ta (destabilization), while that of Ref. [10] predicts a small $(<10 \%)$ increase in Ta (stabilization). This discrepancy may be attributed to the shear-rate dependent rotary diffusivity [Eq. (41)], or to the assumed artificial diffusion and associated boundary conditions for $\boldsymbol{a}^{\prime}$ in Ref. [10], which are absent in the present model.

Figure 11 compares the present model (line) with data from Ref. [11] (markers). The authors of Ref. [11] measured the critical Taylor number [Eq. (40)] for aqueous suspensions of disk-shaped clay particles. Instead of the clay concentration, the authors of Ref. [11] report the corresponding fluid index $n$, which characterizes the effective viscosity of the suspension $v_{\text {eff }} / v \sim \dot{\gamma}^{n-1}$, where $n=1,0<n<1$, and $n=0$ correspond to shear-rate invariance (Newtonian), shear thinning, and yield stress behavior, respectively. The fluid index can be expressed in terms of the slope of the relationship between $v_{\text {eff }} / v$ and Pe:

$$
n=1+\operatorname{Pe} \frac{v}{v_{\text {eff }}} \frac{\partial}{\partial \mathrm{Pe}}\left(\frac{v_{\text {eff }}}{v}\right)
$$

The fluid index for dilute suspensions of disks is found by inserting $v_{\text {eff }}=v\left(1+\alpha_{0} \mathrm{Pe}^{-m}\right)$ into Eq. (42), where $m \approx 5 / 8$ (see Fig. 3), and by using that $\alpha_{0} \mathrm{Pe}^{-m} \ll 1$ :

$$
n=1-m \alpha_{0} \mathrm{Pe}^{-m} \text {. }
$$

The comparison in Fig. 11 concerns clay particles with a disk diameter of $2 b=320 \mathrm{~nm}$ and an aspect ratio of $r_{a}=3.1 \times 10^{-3}$ in a Taylor Couette cell with an inner radius of $R_{1}=9.75 \mathrm{~mm}$ and a radius ratio of: $R_{2} / R_{1}=1.05$, and we focus on the mode, with a spanwise wave number of $k=\pi / \Delta R$. For $r_{a} \rightarrow 0$, the rotary diffusivity equals (see the Appendix)

$$
D_{r}=\frac{9}{16} \frac{k_{B} T}{\eta b^{3}},
$$

which gives $D_{r}=5.5 \times 10^{2} \mathrm{~s}^{-1}$. Here $\eta$ is the dynamic viscosity of water and $k_{B} T$ is the Boltzmann energy. The corresponding Peclet number at a critical Taylor number of $\mathrm{Ta} \approx 42$ is found by combining Eqs. (1), (6), and (44),

$$
\mathrm{Pe}=\mathrm{Ta} \frac{16}{9} \frac{\eta^{2} b^{3}}{\rho k_{B} T} \sqrt{\frac{R_{1}}{\Delta R^{5}}},
$$


which gives $\mathrm{Pe}=1.4$, where $\rho$ is the mass density of water.

Figure 11 shows that with increasing clay concentration or decreasing fluid index $n$, the experimentally measured Ta decreases drastically: up to $75 \%$ for $n=0.05$. The modeled Ta, on the other hand, increases slightly: up to $5 \%$ for $n=0.77$, which corresponds to $\alpha_{0}=1$, which is the maximum value before the numerical solution develops unphysical wiggles.

This discrepancy may be attributed to (hydrodynamic and electrostatic) interactions between the clay particles; see, e.g., Ref. [17]. In the model, these interactions are absent, and at $\mathrm{Pe}=1.4$, the Brownian motion randomizes the particle orientations, resulting in nearly Newtonian behavior. In the semidilute experiments, on the other hand, particle interactions prevent this randomization, promoting the suspension anisotropy [18], which is presumably driving the instability.

\section{CONCLUSIONS}

We have theoretically demonstrated the destabilizing effect of dilute suspensions of disks in Taylor Couette flow (TCF). The instability is driven by anisotropic viscous forces that result from a tilting of the disk normal vector away from the neutral $r$ direction and toward the $z$ direction. This tilting allows the disks to transfer energy from the base flow into azimuthal velocity fluctuations. The wavelength of these fluctuations is smaller than that of the Newtonian instability mode.

The instability mechanism involves an anisotropic microstructure, which requires a sufficiently large Peclet number, Pe $\gg 1$. Available experimental work, however, is concerned with $\mathrm{Pe} \sim 1$ and semidilute concentrations [11]. The experimentally observed instability seems therefore affected by interactions between the disks, which counteract the randomizing effect of the Brownian motion, providing the required anisotropic microstructure [18]. Reproducing the experimental findings of Ref. [11] therefore requires including these interactions in the constitutive equations, which to our knowledge has not yet been done. It is noted that interactions between rods, which have a randomizing effect on the orientations [19], have been modeled as rotary diffusion; see, e.g., Ref. [10].

Finally, we would like to recommend the experimental characterization of TCF instability in dilute suspensions of non-Brownian disks. As indicated by Eq. (45), non-Brownian conditions $(\mathrm{Pe} \gg 1)$ are realizable using large disks and a large solvent viscosity.

\section{ACKNOWLEDGMENT}

We would like to acknowledge financial support from the Engineering and Physical Sciences Research Council of the United Kingdom Grant No. EP/N024915/1.

\section{APPENDIX: MATERIAL FUNCTIONS FOR SPHEROID SUSPENSIONS}

Adopting the notation of Ref. [12], the material functions of Eq. (5) are given by [Eqs. (3.6)-(3.12) in Ref. [12]]

$$
\alpha_{1}=\frac{5}{2} c Q_{1}, \quad \alpha_{2}=-\frac{5 c}{4}\left(3 Q_{2}+4 Q_{3}^{0}\right), \quad \alpha_{3}=5 c Q_{3}^{0},
$$

and

$$
\alpha_{4}=\frac{5}{2} c N
$$

The rotary diffusivity is given by

$$
D_{r}=\frac{k_{B} T}{V_{p} \eta K_{\perp}^{r}},
$$


where $k_{B} T$ is the Boltzmann energy, $\eta=\rho v$ is the solvent dynamic viscosity, and $V_{p}$ is the spheroid volume,

$$
V_{p}=\frac{4 \pi}{3} a b^{2}
$$

Furthermore,

$$
\begin{aligned}
& Q_{1}=\frac{1}{5 \alpha_{\|}^{\prime}}, \quad Q_{2}=\frac{2}{15 \alpha_{\|}^{\prime}}\left(1-\frac{\alpha_{\|}^{\prime \prime}}{\alpha_{\perp}^{\prime \prime}}\right), \quad Q_{3}=\frac{1}{5 \alpha_{\|}^{\prime}}\left[\frac{r_{a}\left(\alpha_{\|}+\alpha_{\perp}\right) \alpha_{\|}^{\prime}}{\left(r_{a}^{2} \alpha_{\|}+\alpha_{\perp}\right) \alpha_{\perp}^{\prime}}-1\right], \\
& Q_{3}^{0}=Q_{3}-\frac{1}{2} B N, \quad N=\frac{2\left(r_{a}^{2}-1\right)}{5\left(r_{a}^{2} \alpha_{\|}+\alpha_{\perp}\right)}, \quad K_{\perp}^{r}=\frac{2\left(r_{a}^{2}+1\right)}{3\left(r_{a}^{2} \alpha_{\|}+\alpha_{\perp}\right)}, \quad B=\frac{r_{a}^{2}-1}{r_{a}^{2}+1}, \\
& \alpha_{\perp}=\frac{r_{a}^{2}}{r_{a}^{2}-1}(1-\beta), \quad \alpha_{\|}=\frac{2}{r_{a}^{2}-1}\left(r_{a}^{2} \beta-1\right), \quad \alpha_{\perp}^{\prime}=\frac{r_{a}}{\left(r_{a}^{2}-1\right)^{2}}\left(r_{a}^{2}+2-3 r_{a}^{2} \beta\right), \\
& \alpha_{\|}^{\prime}=\frac{r_{a}^{2}}{4\left(r_{a}^{2}-1\right)^{2}}\left(3 \beta+2 r_{a}^{2}-5\right), \quad \alpha_{\perp}^{\prime \prime}=\frac{r_{a}^{2}}{\left(r_{a}^{2}-1\right)^{2}}\left[\left(2 r_{a}^{2}+1\right) \beta-3\right], \\
& \alpha_{\|}^{\prime \prime}=\frac{r_{a}^{2}}{4\left(r_{a}^{2}-1\right)^{2}}\left[2 r_{a}^{2}+1-\left(4 r_{a}^{2}-1\right) \beta\right],
\end{aligned}
$$

and

$$
\beta= \begin{cases}\frac{\operatorname{acosh}\left(r_{a}\right)}{r_{a} \sqrt{r_{a}^{2}-1}} & \text { if } r_{a}>1 \\ \frac{\mathrm{acos}\left(r_{a}\right)}{r_{a} \sqrt{1-r_{a}^{2}}} & \text { if } r_{a}<1\end{cases}
$$

[1] G. I. Taylor, Stability of a viscous liquid contained between two rotating cylinders, Philos. Trans. R. Soc., A 223, 289 (1923).

[2] R. G. Larson, E. S. G. Shaqfeh, and S. J. Muller, A purely elastic instability in Taylor-Couette flow, J. Fluid Mech. 218, 573 (1990).

[3] A. Groisman and V. Steinberg, Couette-Taylor Flow in a Dilute Polymer Solution, Phys. Rev. Lett. 77, 1480 (1996).

[4] R. H. Thomas and K. Walters, The stability of elastico-viscous flow between rotating cylinders, part 1, J. Fluid Mech. 18, 33 (1964).

[5] Y. L. Joo and E. S. G. Shaqfeh, The effects of inertia on the viscoelastic Dean and Taylor-Couette flow instabilities with application to coating flows, Phys. Fluids 4, 2415 (1992).

[6] M.-K. Yi and C. Kim, Experimental studies on the Taylor instability of dilute polymer solutions, J. NonNewtonian Fluid Mech. 72, 113 (1997).

[7] B. Martínez-Arias and J. Peixinho, Torque in Taylor-Couette flow of viscoelastic polymer solutions, J. Non-Newtonian Fluid Mech. 247, 221 (2017).

[8] O. Crumeyrolle, I. Mutabazi, and M. Grisel, Experimental study of inertioelastic Couette-Taylor instability modes in dilute and semidilute polymer solutions, Phys. Fluids 14, 1681 (2002).

[9] T. J. Lockett, S. M. Richardson, and W. J. Worraker, The stability of inelastic non-Newtonian fluids in Couette flow between concentric cylinders: A finite-element study, J. Non-Newtonian Fluid Mech. 43, 165 (1992).

[10] V. K. Gupta, R. Sureshkumar, B. Khomami, and J. Azaiez, Centrifugal instability of semidilute nonBrownian fiber suspensions, Phys. Fluids 14, 1958 (2002). 
[11] A. M. Philippe, C. Baravian, M. Jenny, F. Meneau, and L. J. Michot, Taylor-Couette Instability in Anisotropic Clay Suspensions Measured Using Small-Angle X-Ray Scattering, Phys. Rev. Lett. 108, 254501 (2012).

[12] H. Brenner, Rheology of a dilute suspension of axisymmetric Brownian particles, Int. J. Multiphase Flow 1, 195 (1974).

[13] S. G. Advani and C. L. Tucker III, Closure approximations for three-dimensional structure tensors, J. Rheol. 34, 367 (1990).

[14] C. Canuto, M. Y. Hussaini, A. Quarteroni, and A. Thomas, Jr., Spectral Methods in Fluid Dynamics (Springer, Berlin, 2012).

[15] R. C. DiPrima, P. M. Eagles, and B. S. Ng, The effect of radius ratio on the stability of Couette flow and Taylor vortex flow, Phys. Fluids 27, 2403 (1984).

[16] F. Folgar and C. L. Tucker III, Orientation behavior of fibers in concentrated suspensions, J. Reinf. Plast. Compos. 3, 98 (1984).

[17] C. Baravian, D. Vantelon, and F. Thomas, Rheological determination of interaction potential energy for aqueous clay suspensions, Langmuir 19, 8109 (2003).

[18] A. B. D. Brown, S. M. Clarke, P. Convert, and A. R. Rennie, Orientational order in concentrated dispersions of plate-like kaolinite particles under shear, J. Rheol. 44, 221 (2000).

[19] C. A. Stover, D. L. Koch, and C. Cohen, Observations of fibre orientation in simple shear flow of semidilute suspensions, J. Fluid Mech. 238, 277 (1992). 